\title{
ON THE COMPACTNESS OF THE PRODUCT OF HANKEL OPERATORS ON THE SPHERE
}

\author{
JINGBO XIA
}

(Communicated by Joseph A. Ball)

\begin{abstract}
Consider Hankel operators $H_{\varphi}$ and $H_{\psi}$ on the unit sphere in $\mathbf{C}^{n}$. If $n=1$, then a necessary condition for $H_{\varphi}^{*} H_{\psi}$ to be compact is $\lim _{|z| \uparrow 1}\left\|H_{\varphi} k_{z}\right\|\left\|H_{\psi} k_{z}\right\|=0$. We show that when $n \geq 2$, this condition is no longer necessary for $H_{\varphi}^{*} H_{\psi}$ to be compact.
\end{abstract}

\section{INTRODUCTION}

Let $S$ denote the unit sphere $\left\{z \in \mathbf{C}^{n}:|z|=1\right\}$ in $\mathbf{C}^{n}$. Let $\sigma$ be the positive, regular Borel measure on $S$ which is invariant under the orthogonal group $O(2 n)$, i.e., the group of isometries on $\mathbf{C}^{n} \cong \mathbf{R}^{2 n}$ which fix 0 . Furthermore we normalize $\sigma$ such that $\sigma(S)=1$. The Hardy space $H^{2}(S)$ is the norm closure in $L^{2}(S, d \sigma)$ of the collection of polynomials in the complex variables $z_{1}, \ldots, z_{n}$ [3, Section 5.6]. Let $P: L^{2}(S, d \sigma) \rightarrow H^{2}(S)$ be the orthogonal projection. For each $\varphi \in L^{\infty}(S, d \sigma)$, the Toeplitz operator $T_{\varphi}: H^{2}(S) \rightarrow H^{2}(S)$ and the Hankel operator $H_{\varphi}: H^{2}(S) \rightarrow$ $L^{2}(S, d \sigma) \ominus H^{2}(S)$ are respectively defined by the formulas

$$
T_{\varphi} h=P \varphi h \quad \text { and } \quad H_{\varphi} h=(1-P) \varphi h,
$$

$h \in H^{2}(S)$. As usual, let $k_{z}$ denote the normalized reproducing kernel function for $H^{2}(S)$. That is, for each $z \in \mathbf{C}^{n}$ with $|z|<1$, we write

$$
k_{z}(w)=\frac{\left(1-|z|^{2}\right)^{n / 2}}{(1-\langle w, z\rangle)^{n}}, \quad|w| \leq 1 .
$$

The main motivation for this investigation comes from the following sufficient condition for the compactness of $H_{\varphi}^{*} H_{\psi}$ due to D. Zheng.

Theorem 1.1 ([6, Theorem 3]). Let $\varphi$ and $\psi$ be in BMO. Then the operator $H_{\varphi}^{*} H_{\psi}$ is compact if

$$
\lim _{|z| \uparrow 1}\left\|H_{\varphi} k_{z}\right\|\left\|H_{\psi} k_{z}\right\|=0 .
$$

Also see the comments on page 22 of [6]. This raises the obvious

Question 1.2. Is (1.1) a necessary condition for the compactness of $H_{\varphi}^{*} H_{\psi}$ ?

In the case $n=1$, the answer to this question is affirmative. Indeed Zheng proved

Received by the editors November 30, 2006 and, in revised form, February 14, 2007.

2000 Mathematics Subject Classification. Primary 47B07, 47B35.

This work was supported in part by National Science Foundation grant DMS-0456448. 
Theorem 1.3 ([5, Theorem 2]). Suppose that $n=1$. If the operator $H_{\varphi}^{*} H_{\psi}$ is compact, then

$$
\lim _{|z| \uparrow 1}\left\|H_{\varphi} k_{z}\right\|\left\|H_{\psi} k_{z}\right\|=0 .
$$
has

Moreover, for any complex dimension $n$, if $H_{\varphi}^{*} H_{\varphi}$ is compact, then one trivially

$$
\lim _{|z| \uparrow 1}\left\|H_{\varphi} k_{z}\right\|^{2}=0 .
$$

Given these two facts, and the fact that (1.1) is such a natural-looking condition, one might be tempted to "extrapolate" that the answer to Question 1.2 is affirmative for all $n \in \mathbf{N}$. The purpose of this paper is to report that that is not the case. In other words, Theorem 1.3 is actually something of an anomaly; in the case $n \geq 2$, (1.1) is not a necessary condition for the compactness of $H_{\varphi}^{*} H_{\psi}$. More precisely, we will prove

Theorem 1.4. For each complex dimension $n \geq 2$, there exists a pair of functions $\varphi$ and $\psi$ in $L^{\infty}(S, d \sigma)$ such that

$$
\limsup _{|z| \uparrow 1}\left\|H_{\varphi} k_{z}\right\|\left\|H_{\psi} k_{z}\right\|>0
$$

and such that the operator $H_{\varphi}^{*} H_{\psi}$ is compact.

This result tells us something that is somewhat anti-intuitive: while Theorem 1.1 cannot be improved in the case $n=1$, for $n \geq 2$ one should try to prove the compactness of $H_{\varphi}^{*} H_{\psi}$ under a condition that is weaker than (1.1)! This leads to the following question for future investigations.

Question 1.5. For $n \geq 2$, what is a necessary and sufficient condition for the compactness of $H_{\varphi}^{*} H_{\psi}$ ?

The basic idea behind Theorem 1.4 is the following. First of all, the problem can be converted to a problem for the product of Toeplitz operators. That is, if $f$ and $g$ are real valued and have disjoint supports, then $H_{f}^{*} H_{g}=-T_{f} T_{g}$. If there is a positive distance between the supports of $f$ and $g$, then $T_{f} T_{g}$ is compact. Furthermore, if $f$ and $g$ depend only on $\left|z_{1}\right|, \ldots,\left|z_{n-1}\right|$, then $T_{f}$ and $T_{g}$ are diagonal operators with respect to the standard orthonormal basis $\left\{e_{i}: i \in \mathbf{Z}_{+}^{n}\right\}$ in $H^{2}(S)$. Therefore $T_{f} T_{g}$ is a diagonal operator with eigenvalues $\left\langle f e_{i}, e_{i}\right\rangle\left\langle g e_{i}, e_{i}\right\rangle, i \in \mathbf{Z}_{+}^{n}$. Thus in order for $\left\|T_{f} T_{g}\right\|$ to be small, it suffices if one of the two factors $\left|\left\langle f e_{i}, e_{i}\right\rangle\right|,\left|\left\langle g e_{i}, e_{i}\right\rangle\right|$ is small for each $i \in \mathbf{Z}_{+}^{n}$. The fact that we have two factors to manipulate allows us to construct $f$ and $g$ such that $\left\|T_{f} T_{g}\right\|$ is arbitrarily small while $\left\|H_{f} k_{0}\right\|\left\|H_{g} k_{0}\right\|$ has a predetermined lower bound. The desired functions $\varphi$ and $\psi$ are then obtained from a sequence of such $f$ 's, a sequence of such $g$ 's, and Möbius transforms. The lower bound for $\left\|H_{f} k_{0}\right\|\left\|H_{g} k_{0}\right\|$ translates into (1.2), and the smallness of $\left\|T_{f} T_{g}\right\|$ results in the compactness of $H_{\varphi}^{*} H_{\psi}$.

The rest of the paper consists of the details of what we described above. Specifically, Section 2 contains the key step, Lemma 2.2. Section 3 recalls Möbius tranforms and associated unitary operators. The proof of Theorem 1.4 is completed in Section 4.

For the rest of the paper we assume $n \geq 2$. 


\section{Two Toeplitz operators}

Let $Q$ denote the "first quadrant" of the closed unit ball in $\mathbf{R}^{n-1}$. In other words,

$$
Q=\left\{\left(x_{1}, \ldots, x_{n-1}\right) \in \mathbf{R}^{n-1}: x_{1}^{2}+\ldots+x_{n-1}^{2} \leq 1 \text { and } x_{1} \geq 0, \ldots, x_{n-1} \geq 0\right\} .
$$

On the compact set $Q$ we define the measure $d \mu$ by the formula

$$
d \mu\left(x_{1}, \ldots, x_{n-1}\right)=(n-1) ! 2^{n-1} x_{1} \ldots x_{n-1} d x_{1} \ldots d x_{n-1} .
$$

Using the technique described on page 17 of [3], it is straightforward to verify that

$$
\int_{Q} x_{1}^{2 i_{1}} \ldots x_{n-1}^{2 i_{n-1}} d \mu\left(x_{1}, \ldots, x_{n-1}\right)=\frac{(n-1) ! i_{1} ! \ldots i_{n-1} !}{\left(n-1+i_{1}+\ldots+i_{n-1}\right) !}
$$

for all integers $i_{1}, \ldots, i_{n-1}$ in $\mathbf{Z}_{+}$. But for such $i_{1}, \ldots, i_{n-1}$ we also have

$$
\int_{S}\left|z_{1}\right|^{2 i_{1}} \ldots\left|z_{n-1}\right|^{2 i_{n-1}} d \sigma\left(z_{1}, \ldots, z_{n-1}, z_{n}\right)=\frac{(n-1) ! i_{1} ! \ldots i_{n-1} !}{\left(n-1+i_{1}+\ldots+i_{n-1}\right) !}
$$

[3, Proposition 1.4.9]. Hence, by the Stone-Weierstrass approximation theorem, we have

$$
\int_{S} \xi\left(\left|z_{1}\right|, \ldots,\left|z_{n-1}\right|\right) d \sigma\left(z_{1}, \ldots, z_{n-1}, z_{n}\right)=\int_{Q} \xi\left(x_{1}, \ldots, x_{n-1}\right) d \mu\left(x_{1}, \ldots, x_{n-1}\right)
$$

for every $\xi \in C(Q)$.

We will use the usual multi-index notation [3, page 3]. For each $i \in \mathbf{Z}_{+}^{n}$, define

and the function

$$
c_{i}=\left\{\frac{(n-1+|i|) !}{(n-1) ! i !}\right\}^{1 / 2}
$$

$$
e_{i}(z)=c_{i} z^{i}, \quad z \in S
$$

Then $\left\{e_{i}: i \in \mathbf{Z}_{+}^{n}\right\}$ is the standard orthonormal basis for $H^{2}(S)$ [3, Proposition 1.4.9].

For the rest of the paper, we set

$$
\delta=\frac{1}{200(n-1)^{1 / 2}} .
$$

With this fixed $\delta$, we define the subsets $A$ and $B$ of $S$ as follows:

$$
\begin{array}{r}
A=\left\{\left(z_{1}, \ldots, z_{n-1}, z_{n}\right) \in S: \delta<\left|z_{j}\right|<2 \delta \text { for } 1 \leq j \leq n-1\right\} \\
B=\left\{\left(z_{1}, \ldots, z_{n-1}, z_{n}\right) \in S:(n-1)^{-1 / 2}-\delta<\left|z_{j}\right|<(n-1)^{-1 / 2}\right. \\
\text { for } 1 \leq j \leq n-1\} .
\end{array}
$$

Lemma 2.1. (i) Let $f \in C(S)$ be such that $\|f\|_{\infty} \leq 1$. Furthermore, suppose that the support of $f$ is contained in $A$. Then for every $i=\left(i_{1}, \ldots, i_{n-1}, i_{n}\right)$ in $\mathbf{Z}_{+}^{n}$ satisfying the condition

we have $\left|\left\langle f e_{i}, e_{i}\right\rangle\right| \leq 2^{-|i| / 2}$.

$$
i_{1}+\ldots+i_{n-1} \geq i_{n}
$$

(ii) Let $g \in C(S)$ be such that $\|g\|_{\infty} \leq 1$. Furthermore, suppose that the support of $g$ is contained in $B$. Then for every $i=\left(i_{1}, \ldots, i_{n-1}, i_{n}\right)$ in $\mathbf{Z}_{+}^{n}$ satisfying the condition

$$
i_{1}+\ldots+i_{n-1} \leq i_{n}
$$

we have $\left|\left\langle g e_{i}, e_{i}\right\rangle\right| \leq 2^{n-1}(10 / 3)^{-|i| / 4}$. 
Proof. (i) Since $\|f\|_{\infty} \leq 1$ and the support of $f$ is contained in $A$, it is an easy consequence of $(2.1)$ that for every $i=\left(i_{1}, \ldots, i_{n-1}, i_{n}\right) \in \mathbf{Z}_{+}^{n}$ we have

$$
\left|\left\langle f e_{i}, e_{i}\right\rangle\right| \leq \int_{A}\left|e_{i}\right|^{2} d \sigma=c_{i}^{2} \int_{\tilde{A}} x_{1}^{2 i_{1}} \ldots x_{n-1}^{2 i_{n-1}}\left(1-x_{1}^{2}-\ldots-x_{n-1}^{2}\right)^{i_{n}} d \mu\left(x_{1}, \ldots, x_{n-1}\right),
$$

where

$$
\tilde{A}=\left\{\left(x_{1}, \ldots, x_{n-1}\right): \delta<x_{j}<2 \delta \text { for } 1 \leq j \leq n-1\right\},
$$

which is contained in $Q$. By the definition of $\delta, Q$ also contains the set

$$
\tilde{C}=\left\{\left(y_{1}, \ldots, y_{n-1}\right): 4 \delta<y_{j}<5 \delta \text { for } 1 \leq j \leq n-1\right\} .
$$

Also by the definition of $\delta$, if $\left(y_{1}, \ldots, y_{n-1}\right) \in \tilde{C}$, then $y_{1}^{2}+\ldots+y_{n-1}^{2}<1 / 5$. On the other hand, if $\left(x_{1}, \ldots, x_{n-1}\right) \in \tilde{A}$, then $x_{j}+3 \delta>x_{j}+x_{j}=2 x_{j}$ for every $1 \leq j \leq n-1$. Hence

$$
\begin{aligned}
1 & =\int_{S}\left|e_{i}\right|^{2} d \sigma=c_{i}^{2} \int_{Q} y_{1}^{2 i_{1}} \ldots y_{n-1}^{2 i_{n-1}}\left(1-y_{1}^{2}-\ldots-y_{n-1}^{2}\right)^{i_{n}} d \mu\left(y_{1}, \ldots, y_{n-1}\right) \\
& \geq c_{i}^{2} \int_{\tilde{C}} y_{1}^{2 i_{1}} \ldots y_{n-1}^{2 i_{n-1}}(4 / 5)^{i_{n}} d \mu\left(y_{1}, \ldots, y_{n-1}\right) \\
& =(4 / 5)^{i_{n}} c_{i}^{2}(n-1) ! 2^{n-1} \int_{\tilde{C}} y_{1}^{2 i_{1}+1} \ldots y_{n-1}^{2 i_{n-1}+1} d y_{1} \ldots d y_{n-1} \\
& =(4 / 5)^{i_{n}} c_{i}^{2}(n-1) ! 2^{n-1} \int_{\tilde{A}}\left(x_{1}+3 \delta\right)^{2 i_{1}+1} \ldots\left(x_{n-1}+3 \delta\right)^{2 i_{n-1}+1} d x_{1} \ldots d x_{n-1} \\
& \geq(4 / 5)^{i_{n}} c_{i}^{2}(n-1) ! 2^{n-1} \int_{\tilde{A}} 2^{2\left(i_{1}+\ldots+i_{n-1}\right)} x_{1}^{2 i_{1}+1} \ldots x_{n-1}^{2 i_{n-1}+1} d x_{1} \ldots d x_{n-1} \\
& =(4 / 5)^{i_{n}} 2^{2\left(i_{1}+\ldots+i_{n-1}\right)} c_{i}^{2} \int_{\tilde{A}} x_{1}^{2 i_{1}} \ldots x_{n-1}^{2 i_{n-1}} d \mu\left(x_{1}, \ldots, x_{n-1}\right) \\
& \geq(4 / 5)^{i_{n}} 2^{2\left(i_{1}+\ldots+i_{n-1}\right)}\left|\left\langle f e_{i}, e_{i}\right\rangle\right| .
\end{aligned}
$$

If $i_{1}+\ldots+i_{n-1} \geq i_{n}$, then $(4 / 5)^{i_{n}} 2^{2\left(i_{1}+\ldots+i_{n-1}\right)} \geq 2^{i_{1}+\ldots+i_{n-1}} \geq 2^{|i| / 2}$. This proves (i).

(ii) Since $\|g\|_{\infty} \leq 1$ and the support of $g$ is contained in $B$, it is an easy consequence of $(2.1)$ that for every $i=\left(i_{1}, \ldots, i_{n-1}, i_{n}\right) \in \mathbf{Z}_{+}^{n}$ we have

$$
\left|\left\langle g e_{i}, e_{i}\right\rangle\right| \leq \int_{B}\left|e_{i}\right|^{2} d \sigma=c_{i}^{2} \int_{\tilde{B}} x_{1}^{2 i_{1}} \ldots x_{n-1}^{2 i_{n-1}}\left(1-x_{1}^{2}-\ldots-x_{n-1}^{2}\right)^{i_{n}} d \mu\left(x_{1}, \ldots, x_{n-1}\right),
$$

where

(2.3) $\tilde{B}=\left\{\left(x_{1}, \ldots, x_{n-1}\right):(n-1)^{-1 / 2}-\delta<x_{j}<(n-1)^{-1 / 2}\right.$ for $\left.1 \leq j \leq n-1\right\}$,

which is contained in $Q$. By the definition of $\delta, Q$ also contains the set

$\tilde{D}=\left\{\left(y_{1}, \ldots, y_{n-1}\right):(n-1)^{-1 / 2}-6 \delta<y_{j}<(n-1)^{-1 / 2}-5 \delta\right.$ for $\left.1 \leq j \leq n-1\right\}$.

The choice of $\delta$ ensures that if $\left(x_{1}, \ldots, x_{n-1}\right) \in \tilde{B}$, then

$$
\begin{aligned}
& 1-x_{1}^{2}-\ldots-x_{n-1}^{2} \leq 1 / 100, \\
& 1-\left(x_{1}-5 \delta\right)^{2}-\ldots-\left(x_{n-1}-5 \delta\right)^{2} \geq 1 / 30, \quad \text { and } \\
& x_{j}-5 \delta \geq(9 / 10) x_{j} \quad \text { for } 1 \leq j \leq n-1 .
\end{aligned}
$$


Therefore

$$
\begin{aligned}
& 1=\int_{S}\left|e_{i}\right|^{2} d \sigma \geq c_{i}^{2} \int_{\tilde{D}} y_{1}^{2 i_{1}} \ldots y_{n-1}^{2 i_{n-1}}\left(1-y_{1}^{2}-\ldots-y_{n-1}^{2}\right)^{i_{n}} d \mu\left(y_{1}, \ldots, y_{n-1}\right) \\
&= c_{i}^{2}(n-1) ! 2^{n-1} \int_{\tilde{D}} y_{1}^{2 i_{1}+1} \ldots y_{n-1}^{2 i_{n-1}+1}\left(1-y_{1}^{2}-\ldots-y_{n-1}^{2}\right)^{i_{n}} d y_{1} \ldots d y_{n-1} \\
&= c_{i}^{2}(n-1) ! 2^{n-1} \int_{\tilde{B}} \prod_{j=1}^{n-1}\left(x_{j}-5 \delta\right)^{2 i_{j}+1} \cdot\left(1-\sum_{j=1}^{n-1}\left(x_{j}-5 \delta\right)^{2}\right)^{i_{n}} d x_{1} \ldots d x_{n-1} \\
& \geq(9 / 10)^{2\left(i_{1}+\ldots+i_{n-1}\right)+n-1}(10 / 3)^{i_{n}} \\
& \quad \times c_{i}^{2}(n-1) ! 2^{n-1} \int_{\tilde{B}} x_{1}^{2 i_{1}+1} \ldots x_{n-1}^{2 i_{n-1}+1}\left(1-x_{1}^{2}-\ldots-x_{n-1}^{2}\right)^{i_{n}} d x_{1} \ldots d x_{n-1} \\
&=(9 / 10)^{2\left(i_{1}+\ldots+i_{n-1}\right)+n-1}(10 / 3)^{i_{n}} \\
& \quad \times c_{i}^{2} \int_{\tilde{B}} x_{1}^{2 i_{1}} \ldots x_{n-1}^{2 i_{n-1}}\left(1-\sum_{j=1}^{n-1} x_{j}^{2}\right) \\
& \geq 2^{-(n-1)}(9 / 10)^{2\left(i_{1}+\ldots+i_{n-1}\right)}(10 / 3)^{i_{n}}\left|\left\langle g e_{i}, e_{i}\right\rangle\right| .
\end{aligned}
$$

Since $(9 / 10)^{2}(10 / 3)^{1 / 2}>1$, if $i_{n} \geq i_{1}+\ldots+i_{n-1}$, then $(9 / 10)^{2\left(i_{1}+\ldots+i_{n-1}\right)}(10 / 3)^{i_{n}}$ $\geq(10 / 3)^{i_{n} / 2} \geq(10 / 3)^{|i| / 4}$. This completes the proof.

For each $f \in L^{2}(S, d \sigma)$, denote

$$
\operatorname{Var}(f)=\int\left|f-\int f d \sigma\right|^{2} d \sigma .
$$

Lemma 2.2. For any given $\epsilon>0$, there exist real-valued $\tilde{f}, \tilde{g} \in C(Q)$ with $\|\tilde{f}\|_{\infty} \leq$ 1 and $\|\tilde{g}\|_{\infty} \leq 1$ such that the functions $f$ and $g$ defined by the formulas

$f\left(z_{1}, \ldots, z_{n-1}, z_{n}\right)=\tilde{f}\left(\left|z_{1}\right|, \ldots,\left|z_{n-1}\right|\right) \quad$ and $\quad g\left(z_{1}, \ldots, z_{n-1}, z_{n}\right)=\tilde{g}\left(\left|z_{1}\right|, \ldots,\left|z_{n-1}\right|\right)$,

$\left(z_{1}, \ldots, z_{n-1}, z_{n}\right) \in S$, have the following properties:

$(\alpha)$ The support of $f$ is contained in $A$ and the support of $g$ is contained in $B$.

( $\beta) \operatorname{Var}(f) \geq(1 / 3) \delta^{2(n-1)}$ and $\operatorname{Var}(g) \geq(1 / 3) \delta^{2(n-1)}$.

$(\gamma)\left\|T_{f} T_{g}\right\| \leq \epsilon$.

Proof. Given $\epsilon>0$, let $N \in \mathbf{N}$ be such that $2^{n-1}(10 / 3)^{-N / 4} \leq \epsilon$. We first show that there is a real-valued $\tilde{f} \in C(Q)$ with $\|\tilde{f}\|_{\infty} \leq 1$ such that the function $f$ defined by (2.4) has the following properties:

(i) The support of $f$ is contained in $A$.

(ii) $\left|\left\langle f e_{i}, e_{i}\right\rangle\right| \leq \epsilon$ if $|i| \leq N$.

(iii) $\operatorname{Var}(f) \geq(1 / 3) \delta^{2(n-1)}$.

To construct such an $f$, let $F=\left\{i \in \mathbf{Z}_{+}^{n}:|i| \leq N\right\}$ and let $d m_{n-1}$ denote the standard Lebesgue measure on $\mathbf{R}^{n-1}$. For each $i=\left(i_{1}, \ldots, i_{n-1}, i_{n}\right)$ in $F$, define the function

$$
u_{i}\left(x_{1}, \ldots, x_{n-1}\right)=c_{i}^{2}(n-1) ! 2^{n-1} x_{1}^{2 i_{1}+1} \ldots x_{n-1}^{2 i_{n-1}+1}\left(1-x_{1}^{2}-\ldots-x_{n-1}^{2}\right)^{i_{n}}
$$

on $Q$. Since each $u_{i}$ is continuous and since $\operatorname{card}(F)<\infty$, for the given $\epsilon$ we can decompose the cube $\tilde{A}$ defined by (2.2) as the union of a finite family of pairwise 
disjoint subcubes $\left\{\tilde{A}_{j}: j \in J\right\}$ such that for each $j \in J$ and each $i \in F$, we have

$$
\left|u_{i}(x)-u_{i}(y)\right| \leq \epsilon \quad \text { for all } x, y \in \tilde{A}_{j} .
$$

Now, for each $j \in J$, it is elementary to construct a real-valued function $\tilde{f}_{j} \in C(Q)$ which has the following properties:

(a) The support of $\tilde{f}_{j}$ is contained in the interior of $\tilde{A}_{j}$.

(b) $-1 \leq \tilde{f}_{j} \leq 1$.

(c) $m_{n-1}\left(\left\{x: \tilde{f}_{j}(x)=1\right\}\right) \geq(1 / 3) m_{n-1}\left(\tilde{A}_{j}\right)$.

(d) $m_{n-1}\left(\left\{x: \tilde{f}_{j}(x)=-1\right\}\right) \geq(1 / 3) m_{n-1}\left(\tilde{A}_{j}\right)$.

(e) $\int_{\tilde{A}_{j}} \tilde{f}_{j} d m_{n-1}=0$.

Define $\tilde{f}=\sum_{j \in J} \tilde{f}_{j}$. Then $\tilde{f} \in C(Q),-1 \leq f \leq 1$, and the support of $\tilde{f}$ is contained in $\tilde{A}$. Hence the support of $f$ is contained in $A$, verifying (i).

To verify (ii), apply (2.1), (2.5) and (e). For each $i \in F$ we have

$$
\left\langle f e_{i}, e_{i}\right\rangle=\int_{Q} u_{i} \tilde{f} d m_{n-1}=\sum_{j \in J} \int_{\tilde{A}_{j}} u_{i} \tilde{f}_{j} d m_{n-1}=\sum_{j \in J} \int_{\tilde{A}_{j}}\left(u_{i}-u_{i}\left(a_{j}\right)\right) \tilde{f}_{j} d m_{n-1},
$$

where $a_{j}$ is any chosen point in $\tilde{A}_{j}$. Combining this with (2.6), we conclude that

$$
\left|\left\langle f e_{i}, e_{i}\right\rangle\right| \leq \sum_{j \in J} m_{n-1}\left(\tilde{A}_{j}\right) \epsilon=m_{n-1}(\tilde{A}) \epsilon \leq \epsilon,
$$

$i \in F$. To prove (iii), observe that (c) and (d) together give us the estimate

$$
\int_{\tilde{A}}|\tilde{f}-c|^{2} d m_{n-1} \geq \sum_{j \in J} \frac{1}{3} m_{n-1}\left(\tilde{A}_{j}\right)=\frac{1}{3} m_{n-1}(\tilde{A})=\frac{1}{3} \delta^{n-1}
$$

for every $c \in \mathbf{C}$. By $(2.1)$ and the fact that $x_{1} \ldots x_{n-1} \geq \delta^{n-1}$ if $\left(x_{1}, \ldots, x_{n-1}\right) \in \tilde{A}$, we have

$$
\int|f-c|^{2} d \sigma=\int_{Q}|\tilde{f}-c|^{2} d \mu \geq \delta^{n-1} \int_{\tilde{A}}|\tilde{f}-c|^{2} d m_{n-1} \geq \frac{1}{3} \delta^{2(n-1)} .
$$

This proves (iii) and complets the construction of $f$.

To construct $\tilde{g}$, consider the cube $\tilde{B}$ defined by (2.3). It is elementary that there is a real-valued $\tilde{g} \in C(Q)$ which has the following properties:

(1) The support of $\tilde{g}$ is contained in the interior of $\tilde{B}$.

(2) $-1 \leq \tilde{g} \leq 1$.

(3) $m_{n-1}(\{x: \tilde{g}(x)=1\}) \geq(1 / 3) m_{n-1}(\tilde{B})$.

(4) $m_{n-1}(\{x: \tilde{g}(x)=-1\}) \geq(1 / 3) m_{n-1}(\tilde{B})$.

Then (3) and (4) together imply that

$$
\int_{\tilde{B}}|\tilde{g}-c|^{2} d m_{n-1} \geq \frac{1}{3} m_{n-1}(\tilde{B})=\frac{1}{3} \delta^{n-1}
$$

for every $c \in \mathbf{C}$. By $(2.3)$ and the definition of $\delta$, if $\left(x_{1}, \ldots, x_{n-1}\right) \in \tilde{B}$, then $x_{1} \ldots x_{n-1}$ $\geq\left\{(n-1)^{-1 / 2}-\delta\right\}^{n-1} \geq \delta^{n-1}$. Thus it follows from (2.4) and (2.1) that

$$
\int|g-c|^{2} d \sigma=\int_{Q}|\tilde{g}-c|^{2} d \mu \geq \delta^{n-1} \int_{\tilde{B}}|\tilde{g}-c|^{2} d m_{n-1} \geq \frac{1}{3} \delta^{2(n-1)} .
$$

This establishes $(\alpha)$ and $(\beta)$. 
To prove $(\gamma)$, note that $(2.4)$ implies $\left\langle f e_{i}, e_{i^{\prime}}\right\rangle=0=\left\langle g e_{i}, e_{i^{\prime}}\right\rangle$ for all $i \neq i^{\prime}$ in $\mathbf{Z}_{+}^{n}$. Thus the Toeplitz operators $T_{f}$ and $T_{g}$ are diagonal operators with respect to the orthonormal basis $\left\{e_{i}: i \in \mathbf{Z}_{+}^{n}\right\}$. Consequently

$$
T_{f} T_{g}=\sum_{i \in \mathbf{Z}_{+}^{n}}\left\langle f e_{i}, e_{i}\right\rangle\left\langle g e_{i}, e_{i}\right\rangle e_{i} \otimes e_{i} .
$$

By Lemma 2.1, $\left|\left\langle f e_{i}, e_{i}\right\rangle\left\langle g e_{i}, e_{i}\right\rangle\right| \leq 2^{n-1}(10 / 3)^{-|i| / 4}$ for every $i \in \mathbf{Z}_{+}^{n}$. By the choice of $N$, this gives us $\left|\left\langle f e_{i}, e_{i}\right\rangle\left\langle g e_{i}, e_{i}\right\rangle\right| \leq \epsilon$ in the case $|i| \geq N$. But when $|i| \leq N$, it follows from property (ii) for $f$ that $\left|\left\langle f e_{i}, e_{i}\right\rangle\left\langle g e_{i}, e_{i}\right\rangle\right| \leq\left|\left\langle f e_{i}, e_{i}\right\rangle\right| \leq \epsilon$. Hence $\left\|T_{f} T_{g}\right\| \leq \epsilon$.

\section{MÖBIUS TRANSFORM}

For each $z \in \mathbf{C}^{n}$ with $0<|z|<1$, define the Möbius transform

$$
\varphi_{z}(w)=\frac{1}{1-\langle w, z\rangle}\left\{z-\frac{\langle w, z\rangle}{|z|^{2}} z-\left(1-|z|^{2}\right)^{1 / 2}\left(w-\frac{\langle w, z\rangle}{|z|^{2}} z\right)\right\}, \quad|w| \leq 1 .
$$

Then $\varphi_{z}$ is an involution, i.e., $\varphi_{z} \circ \varphi_{z}=i d$ [3, Theorem 2.2.2]. Recall that the formula

$$
\left(U_{z} f\right)(w)=f\left(\varphi_{z}(w)\right) k_{z}(w)
$$

defines a unitary operator on $L^{2}(S, d \sigma)$ with the property $\left[U_{z}, P\right]=0[4$, Section 6]. Therefore for any $f \in L^{\infty}(S, d \sigma),\left\|H_{f \circ \varphi_{z}} k_{z}\right\|=\left\|(1-P) U_{z} f\right\|=\|(1-P) f\|$. If $f$ is a real-valued function, then $2\|(1-P) f\|^{2} \geq \operatorname{Var}(f)[4,(6.3)]$. Thus we conclude that

$$
\left\|H_{f \circ \varphi_{z}} k_{z}\right\|^{2} \geq \frac{1}{2} \operatorname{Var}(f)
$$

for every real-valued $f \in L^{\infty}(S, d \sigma)$.

Recall that the formula $d(u, v)=|1-\langle u, v\rangle|^{1 / 2}, u, v \in S$, defines a metric on $S$ [3, page 66]. For $u \in S$ and $a>0$, let $B(u, a)$ denote the open ball with respect to the metric $d$. That is, we write

$$
B(u, a)=\left\{v \in S:|1-\langle u, v\rangle|^{1 / 2}<a\right\} .
$$

Lemma 3.1. Let $0<a<1$. If $1-\left(a^{4} / 4\right)<r<1$, then for every $u \in S$ we have

$$
\varphi_{r u}(S \backslash B(u, a)) \subset B(u, a) .
$$

Proof. It is easy to see that $\varphi_{r u}(-u)=u$ if $0<r<1$ and $u \in S$. For such $r$ and $u$, it follows from [3, Theorem 2.2.2] that

$$
1-\left\langle\varphi_{r u}(w), u\right\rangle=1-\left\langle\varphi_{r u}(w), \varphi_{r u}(-u)\right\rangle=\frac{(1-r)(1+\langle w, u\rangle)}{1-r\langle w, u\rangle} .
$$

It is elementary that if $|c| \leq 1$ and $0<r<1$, then $2|1-r c| \geq|1-c|$. Hence

$$
\left|1-\left\langle\varphi_{r u}(w), u\right\rangle\right| \leq \frac{4(1-r)}{|1-\langle w, u\rangle|}
$$

for $0<r<1$ and $w, u \in S$. Therefore if $1-r<a^{4} / 4$ and $|1-\langle w, u\rangle| \geq a^{2}$, then $\left|1-\left\langle\varphi_{r u}(w), u\right\rangle\right|<a^{2}$. That is, if $1-\left(a^{4} / 4\right)<r<1$ and $w \in S \backslash B(u, a)$, then $\varphi_{r u}(w) \in B(u, a)$. 


\section{Proof of Theorem 1.4}

Let $A, B$ be the same as in Section 2. Then the open set $V=S \backslash(\bar{A} \cup \bar{B})$ is obviously not empty. Thus there exist a sequence of points $\left\{u_{j}\right\}_{j=1}^{\infty}$ in $V$ and a sequence of positive numbers $\left\{a_{j}\right\}_{j=1}^{\infty}$ with $\lim _{j \rightarrow \infty} a_{j}=0$ such that $B\left(u_{j}, a_{j}\right) \subset V$ for every $j$ and

$$
B\left(u_{j}, 2 a_{j}\right) \cap B\left(u_{j^{\prime}}, 2 a_{j^{\prime}}\right)=\emptyset \quad \text { if } j \neq j^{\prime} .
$$

For each $j \in \mathbf{N}$, pick an $r_{j} \in\left(1-\left(a_{j}^{4} / 4\right), 1\right)$. Then $\lim _{j \rightarrow \infty} r_{j}=1$. Define $z(j)=$ $r_{j} u_{j}, j \in \mathbf{N}$. Then Lemma 3.1 tells us that

$$
\varphi_{z(j)}\left(S \backslash B\left(u_{j}, a_{j}\right)\right) \subset B\left(u_{j}, a_{j}\right)
$$

for every $j$.

By Lemma 2.2, for each $j \in \mathbf{N}$ there exist real-valued $f_{j}, g_{j} \in C(S)$ such that

(1) $\left\|f_{j}\right\|_{\infty} \leq 1$ and $\left\|g_{j}\right\|_{\infty} \leq 1$;

(2) the support of $f_{j}$ is contained in $A$ and the support of $g_{j}$ is contained in $B$;

(3) $\operatorname{Var}\left(f_{j}\right) \geq(1 / 3) \delta^{2(n-1)}$ and $\operatorname{Var}\left(g_{j}\right) \geq(1 / 3) \delta^{2(n-1)}$;

(4) $\left\|T_{f_{j}} T_{g_{j}}\right\| \leq 2^{-j}$.

By (4.2) and the fact that $B\left(u_{j}, a_{j}\right) \subset V=S \backslash(\bar{A} \cup \bar{B})$, the supports of $f_{j} \circ \varphi_{z(j)}$ and $g_{j} \circ \varphi_{z(j)}$ are contained in $B\left(u_{j}, a_{j}\right)$. Combining this with (4.1), we have

$$
f_{j} \circ \varphi_{z(j)} \cdot f_{j^{\prime}} \circ \varphi_{z\left(j^{\prime}\right)}=0=g_{j} \circ \varphi_{z(j)} \cdot g_{j^{\prime}} \circ \varphi_{z\left(j^{\prime}\right)} \quad \text { if } j \neq j^{\prime} .
$$

Since $f_{j} g_{j}=0$, we also have

$$
f_{j} \circ \varphi_{z(j)} \cdot g_{j^{\prime}} \circ \varphi_{z\left(j^{\prime}\right)}=0 \text { for all } j, j^{\prime} \in \mathbf{N} .
$$

Denote $c=(1 / 6) \delta^{2(n-1)}$. Since $f_{j}, g_{j}$ are real-valued, (3.2) tells us that

$$
\left\|H_{f_{j} \circ \varphi_{z(j)}} k_{z(j)}\right\|\left\|H_{g_{j} \circ \varphi_{z(j)}} k_{z(j)}\right\| \geq \frac{1}{2}\left\{\operatorname{Var}\left(f_{j}\right) \operatorname{Var}\left(g_{j}\right)\right\}^{1 / 2} \geq c,
$$

$j \in \mathbf{N}$.

It is well known that $\sigma(B(u, a)) \leq A_{0} a^{2 n}\left[3\right.$, Proposition 5.1.4]. Since $\left\|f_{j}\right\|_{\infty} \leq 1$ and $\left\|g_{j}\right\|_{\infty} \leq 1$ and the supports of $f_{j} \circ \varphi_{z(j)}$ and $g_{j} \circ \varphi_{z(j)}$ are contained in $B\left(u_{j}, a_{j}\right)$, we have

$$
\lim _{j \rightarrow \infty}\left\|M_{f_{j} \circ \varphi_{z(j)}} h\right\|=0 \quad \text { and } \quad \lim _{j \rightarrow \infty}\left\|M_{g_{j} \circ \varphi_{z(j)}} h\right\|=0
$$

for every $h \in L^{2}(S, d \sigma)$. By (4.1) and a trivial estimate using the Cauchy integral formula for $P$ [3, Section 3.2],

$$
\lim _{j \rightarrow \infty}\left\|M_{f_{j} \circ \varphi_{z(j)}} P M_{g_{\nu} \circ \varphi_{z(\nu)}}\right\|=0 \quad \text { and } \quad \lim _{j \rightarrow \infty}\left\|M_{f_{\nu} \circ \varphi_{z(\nu)}} P M_{g_{j} \circ \varphi_{z(j)}}\right\|=0
$$

for every $\nu \in \mathbf{N}$. Since $f_{\nu} \circ \varphi_{z(\nu)}, g_{\nu} \circ \varphi_{z(\nu)} \in C(S)$, the Hankel operators $H_{f_{\nu} \circ \varphi_{z(\nu)}}$ and $H_{g_{\nu} \circ \varphi_{z(\nu)}}$ are compact. Therefore for every $\nu \in \mathbf{N}$ we also have

$$
\lim _{j \rightarrow \infty}\left\|H_{f_{\nu} \circ \varphi_{z(\nu)}} k_{z(j)}\right\|=0 \quad \text { and } \quad \lim _{j \rightarrow \infty}\left\|H_{g_{\nu} \circ \varphi_{z(\nu)}} k_{z(j)}\right\|=0 .
$$


Using (4.6), (4.7), (4.8) and a standard induction argument, we can select a strictly increasing sequence of natural numbers $j_{1}<\ldots<j_{m}<\ldots$ such that the inequalities

$$
\begin{array}{r}
\sum_{i=1}^{m-1}\left(\left\|M_{f_{j_{m}} \circ \varphi_{z\left(j_{m}\right)}} k_{z\left(j_{i}\right)}\right\|+\left\|M_{g_{j_{m}} \circ \varphi_{z\left(j_{m}\right)}} k_{z\left(j_{i}\right)}\right\|\right) \leq 2^{-m}, \\
\sum_{i=1}^{m-1}\left(\left\|M_{f_{j_{m}} \circ \varphi_{\left.z_{(j m}\right)}} P M_{g_{j_{i}} \circ \varphi_{z\left(j_{i}\right)}}\right\|+\left\|M_{f_{j_{i}} \circ \varphi_{z\left(j_{i}\right)}} P M_{g_{j_{m}} \circ \varphi_{z\left(j_{m}\right)}}\right\|\right) \leq 2^{-m}, \\
\sum_{i=1}^{m-1}\left(\left\|H_{f_{j_{i}} \circ \varphi_{z\left(j_{i}\right)}} k_{z\left(j_{m}\right)}\right\|+\left\|H_{g_{j_{i}} \circ \varphi_{z\left(j_{i}\right)}} k_{z\left(j_{m}\right)}\right\|\right) \leq 2^{-m}
\end{array}
$$

hold for every $m \geq 2$.

To prove Theorem 1.4, we define

$$
\varphi=\sum_{m=1}^{\infty} f_{j_{m}} \circ \varphi_{z\left(j_{m}\right)} \quad \text { and } \quad \psi=\sum_{m=1}^{\infty} g_{j_{m}} \circ \varphi_{z\left(j_{m}\right)}
$$

By (4.3) and the fact that $\left\|f_{j}\right\|_{\infty} \leq 1$ and $\left\|g_{j}\right\|_{\infty} \leq 1$, we have $\|\varphi\|_{\infty} \leq 1$ and $\|\psi\|_{\infty} \leq 1$. For each $m \geq 2$, it follows from (4.11) and (4.9) that

$$
\begin{aligned}
\left\|H_{\varphi} k_{z\left(j_{m}\right)}\right\| \geq & \left\|H_{f_{j_{m}} \circ \varphi_{z\left(j_{m}\right)}} k_{z\left(j_{m}\right)}\right\|-\sum_{i=1}^{m-1}\left\|H_{f_{j_{i}} \circ \varphi_{z\left(j_{i}\right)}} k_{z\left(j_{m}\right)}\right\| \\
& -\sum_{i=m+1}^{\infty}\left\|H_{f_{j_{i}} \circ \varphi_{z\left(j_{i}\right)}} k_{z\left(j_{m}\right)}\right\| \\
\geq & \left\|H_{f_{j_{m}} \circ \varphi_{z\left(j_{m}\right)}} k_{z\left(j_{m}\right)}\right\|-2^{-m}-\sum_{i=m+1}^{\infty} 2^{-i} \\
= & \left\|H_{f_{j_{m}} \circ \varphi_{z\left(j_{m}\right)}} k_{z\left(j_{m}\right)}\right\|-2^{-m+1} .
\end{aligned}
$$

Similarly, $\left\|H_{\psi} k_{z\left(j_{m}\right)}\right\| \geq\left\|H_{g_{j_{m}} \circ \varphi_{z\left(j_{m}\right)}} k_{z\left(j_{m}\right)}\right\|-2^{-m+1}$. Combining this with (4.5), we have

$$
\begin{aligned}
\left\|H_{\varphi} k_{z\left(j_{m}\right)}\right\|\left\|H_{\psi} k_{z\left(j_{m}\right)}\right\| & \geq\left\|H_{f_{j_{m}} \circ \varphi_{z\left(j_{m}\right)}} k_{z\left(j_{m}\right)}\right\|\left\|H_{g_{j_{m}} \circ \varphi_{z\left(j_{m}\right)}} k_{z\left(j_{m}\right)}\right\|-2^{-m+2} \\
& \geq c-2^{-m+2}
\end{aligned}
$$

for $m \geq 2$. Since $\left|z\left(j_{m}\right)\right|=r_{j_{m}}$ and $\lim _{m \rightarrow \infty} r_{j_{m}}=1$, this proves (1.2).

To prove that $H_{\varphi}^{*} H_{\psi}$ is compact, observe that (4.4) gives us $\varphi \psi=0$. Thus $H_{\varphi}^{*} H_{\psi}=-T_{\bar{\varphi}} T_{\psi}=-T_{\varphi} T_{\psi}$. Hence it suffices to show that $T_{\varphi} T_{\psi}$ is compact. By (4.4) and the fact that $f_{j}, g_{j^{\prime}}$ are continuous, the operator $T_{f_{j} \circ \varphi_{z(j)}} T_{g_{j^{\prime}} \circ \varphi_{z\left(j^{\prime}\right)}}$ is compact for all $j, j^{\prime} \in \mathbf{N}$. Thus, by (4.12), to prove that $T_{\varphi} T_{\psi}$ is compact, it suffices to show that

$$
\sum_{\ell=1}^{\infty} \sum_{m=1}^{\infty}\left\|T_{f_{j_{\ell}} \circ \varphi_{z\left(j_{\ell}\right)}} T_{g_{j_{m}} \circ \varphi_{z\left(j_{m}\right)}}\right\|<\infty
$$


We write the above sum as $X+Y$, where

$$
\begin{aligned}
& X=\sum_{m=1}^{\infty}\left\|T_{f_{j_{m}} \circ \varphi_{z\left(j_{m}\right)}} T_{g_{j_{m}} \circ \varphi_{z\left(j_{m}\right)}}\right\|, \\
& Y=\sum_{i=1}^{\infty} \sum_{m=1}^{\infty}\left(\left\|T_{f_{j_{m+i}} \circ \varphi_{z\left(j_{m+i}\right)}} T_{g_{j_{m}} \circ \varphi_{\left.z_{(j m}\right)}}\right\|+\left\|T_{f_{j_{m}} \circ \varphi_{z\left(j_{m}\right)}} T_{\left.g_{j_{m+i}} \circ \varphi_{z\left(j_{m}+i\right.}\right)}\right\|\right) .
\end{aligned}
$$

By (4.10), we have $Y \leq \sum_{i=1}^{\infty} \sum_{m=1}^{\infty} 2^{-(m+i)}<\infty$. Recalling (3.1), we have

$$
U_{z(j)} T_{f_{j}} T_{g_{j}} U_{z(j)}^{*}=T_{f_{j} \circ \varphi_{z(j)}} T_{g_{j} \circ \varphi_{z(j)}} .
$$

Hence $\left\|T_{f_{j} \circ \varphi_{z(j)}} T_{g_{j} \circ \varphi_{z(j)}}\right\|=\left\|T_{f_{j}} T_{g_{j}}\right\| \leq 2^{-j}$, which leads to the conclusion $X<\infty$.

This proves (4.13) and completes the proof of Theorem 1.4.

\section{REFERENCES}

[1] L. Coburn, Singular integral operators and Toeplitz operators on odd spheres, Indiana Univ. Math. J. 23 (1973/74), 433-439. MR0322595 (48:957)

[2] A. Davie and N. Jewell, Toeplitz operators in several complex variables, J. Funct. Anal. 26 (1977), 356-368. MR0461195 (57:1180)

[3] W. Rudin, Function theory in the unit ball of $\mathbf{C}^{n}$, Springer-Verlag, New York, 1980. MR601594 (82i:32002)

[4] J. Xia, Bounded functions of vanishing mean oscillation on compact metric spaces, J. Funct. Anal. 209 (2004), 444-467. MR2044231 (2005d:46106)

[5] D. Zheng, The distribution function inequality and products of Toeplitz operators and Hankel operators, J. Funct. Anal. 138 (1996), 477-501. MR1395967 (97e:47040)

[6] D. Zheng, Toeplitz operators and Hankel operators on the Hardy space of the unit sphere, J. Funct. Anal. 149 (1997), 1-24. MR1471097 (98m:47038)

Department of Mathematics, State University of New York at Buffalo, Buffalo, NEW YORK 14260

E-mail address: jxia@acsu.buffalo.edu 